\title{
Retalho do músculo reto abdominal transverso autonomizado em pacientes obesas e fumantes
}

\author{
Delayed transverse rectus abdominis myocutaneous breast reconstruction in obese \\ and smoker patient
}

\section{RESUMO}

Introdução: A reconstrução mamária foi difundida no fim da década de 1970 e no início da de 1980 . Nas reconstruções mamárias realizadas por meio do retalho do músculo reto abdominal transverso (TRAM), principalmente em pacientes obesas e fumantes, ocorriam necroses parciais ou totais. Estudos experimentais contribuíram muito para minimizar a necrose, que chegou a cerca de $16 \%$. Porém, nas obesas e fumantes, continuava alarmante o índice de necroses $(75 \%)$. Alguns autores começaram a realizar embolização ou laqueadura das epigástricas inferiores. Objetivo: O objetivo deste trabalho foi apresentar como fazer a autonomização e seus resultados. Método: Estudo retrospectivo de 13 pacientes, nas quais se utilizou a técnica referida. Foram analisadas por meio dos métodos de Student e Tukey, apresentando sua significância. Resultados: Um paciente apresentou epidermólise. Discussão: Dessa forma, a autonomização propicia uma série de fenômenos que resultam, na maioria das vezes, após período de 10 a 21 dias, no aumento da vascularização local. Conclusão: As táticas cirúrgicas de autonomizar o TRAM e realizar a transposição após 15 dias mostraram-se eficazes. Palavras-chave: mamoplastia; reto do abdome; retalhos cirúrgicos; obesidade; hábito de fumar.

\begin{abstract}
Introduction: Post-Mastectomy Breast reconstruction became popular in the late 1970s and early 1980s. At these surgeries through Transverse Rectus Abdominus Myocutaneous flap (TRAM), mainly in obese and smoking patients, it was demonstrated areas of partial or total necrosis. Experimental studies contributed in many ways to minimize these complications to $16 \%$. But the necrosis occurrence was still high in obese and smoking patients (75\%). A few authors began to do embolization or ligature at inferior epigastric plexus. Objective: This study aim is to show a way to make surgical delay of TRAM flap and its results. Methods: Retrospective study from 13 patients, in which this technic was performed. They were analyzed through Student and Tukey methods, presenting its statistical significance Results: One single patient presented epidermolysis. Discussion: The flap delay creates several local exchanges that results, usually after 10 to 21 days, at the increase of local vascular structures. Conclusion: Surgical delay of the TRAM flap as a surgical tactic and its definitive reconstruction after 15 days has been effective.
\end{abstract}

Keywords: mammoplasty; rectus abdominis; surgical flaps; obesity; smoking.

\section{INTRODUÇÃ̃}

Tanzini apresentou a técnica de reconstrução mamária através do músculo grande dorsal em 1896. Bostwick revitalizou essa técnica nos fins da década de 1970. A reconstrução mamária por meio do retalho do músculo reto abdominal transverso (TRAM, em inglês: Trans Rectal Abdominal Muscle) surgiu na década de 1980. Nos eventos das Sociedades de Mastologia e da Cirurgia Plástica, ocorriam inúmeras discussões sobre a vascularização do retalho retoabdominal, realizada pelas artérias epigástricas superiores e inferiores. Como esse retalho é ilhado, abrangendo um leque de pele e tecido cutâneo, quando elevado, sua vascularização passa a ser, somente, pela artéria epigástrica superior, podendo não suprir totalmente o retalho. Assim, havia muitas opiniões sobre realizar a cirurgia do retalho ilhado com um só pedículo ou dois dos músculos retoabdominais. O retalho ilhado foi dividido em quatro zonas em seu suprimento sanguíneo, através da artéria epigástrica superior; além do perigo da ocorrência de necroses. ${ }^{1-4}$

${ }^{1}$ Pontifícia Universidade Católica de São Paulo (PUC-SP), Faculdade de Ciências Médicas e da Saúde - Sorocaba (SP), Brasil. Contato: hagonella@ hotmail.com

Recebido em 04/11/2015. Aceito para publicação em 22/03/2016. 
Portanto, por ser mais seguro, sempre utilizamos dois pedículos. Através dos anos e da curva de aprendizado dessa cirurgia, a porcentagem de complicações e necroses reduziu para $16 \%{ }^{1}$ No entanto, apesar de todos os esforços, em pacientes obesas, tabagistas e portadoras de cicatrizes abdominais o risco ainda é alto (necroses parciais e totais), cerca de $75 \% .^{2}$

Estudos experimentais sobre a vascularização dos vários retalhos miocutâneos e seu território vascular, principalmente do plexo da epigástrica inferior, contribuíram consideravelmente para melhores resultados. ${ }^{3-7}$ Alguns trabalhos utilizaram fármacos para avaliar a perfusão e a necrose desses retalhos. ${ }^{8}$

Assim, para efetuar uma maior vascularização nesses retalhos, alguns cirurgiões começaram a realizar a "flap delay" (autonomização) ${ }^{7,9-10}$ em referência ao chamado "delay phenomenon". ${ }^{11-13}$ Outros realizaram a embolização ${ }^{14,15} \mathrm{e}$ alguns, a laqueadura (por meio de laparoscopia) ${ }^{16}$ da artéria epigástrica inferior. Como não existe uma explicação razoável de como a autonomização é realizada, este trabalho teve o objetivo de relatar como e quando utilizamos a autonomização do TRAM e seus efeitos.

\section{MATERIAL E MÉTODO}

Estudo retrospectivo em 13 pacientes, com idades variando de 39 a 67 anos (média de 51,69) e índice de massa corpórea (IMC) entre 26,57 e 31,3 (média=28,295), nas quais foram realizados retalhos TRAM autonomizados. O retalho, planejado e marcado, foi incisado em toda a amplitude da pele e do subcutâneo, acima da aponeurose. Após a identificação da área do plexo epigástrico inferior, a aponeurose foi incisada cerca de 2 a $3 \mathrm{~cm}$ e foi realizada a ligadura dos vasos, bilateralmente. Encerramos a primeira etapa apenas com a síntese da pele. $\mathrm{O}$ procedimento durou entre 35 e 55 minutos e as pacientes receberam alta hospitalar após 12 horas. Retornaram após 15 dias para a realização da transposição do TRAM.

\section{RESULTADOS}

Verificamos um caso de necrose parcial e com resolução completa após tratamento clínico com curativos. Nenhuma paciente apresentou qualquer complicação maior após o procedimento (Figuras 1, 2 e 3 ).

Os dados foram analisados com o teste $t$ de Student ( ignificância=1,771) e com o teste de Tukey $(=1,356)$.

\section{DISCUSSÃO}

A nutrição celular depende da irrigação vascular por meio da pressão arterial, variando de 2 a $120 \mathrm{mmHg}$, dependendo da sua localização. Ao realizar-se um retalho, essa pressão arterial cai para 1 a $2 \mathrm{mmHg}$, suficiente para a sua sobrevida. ${ }^{8}$ Exemplificando: a região do retalho miocutâneo do músculo grácil está próxima de $85 \mathrm{mmHg}$, porém, quando este retalho é elevado, cai para $2 \mathrm{mmHg}$, permanecendo a sua vascularização viável. No entanto, quando confeccionado um retalho espesso, como em pacientes obesos, o fluxo sanguíneo, que cai para 1 a $2 \mathrm{mmHg}$, é insuficiente para suprir toda a nutrição celular do retalho, o que pode ocasionar necrose parcial ou total. Nesses pacientes, táticas cirúrgicas como a autonomização são essenciais.
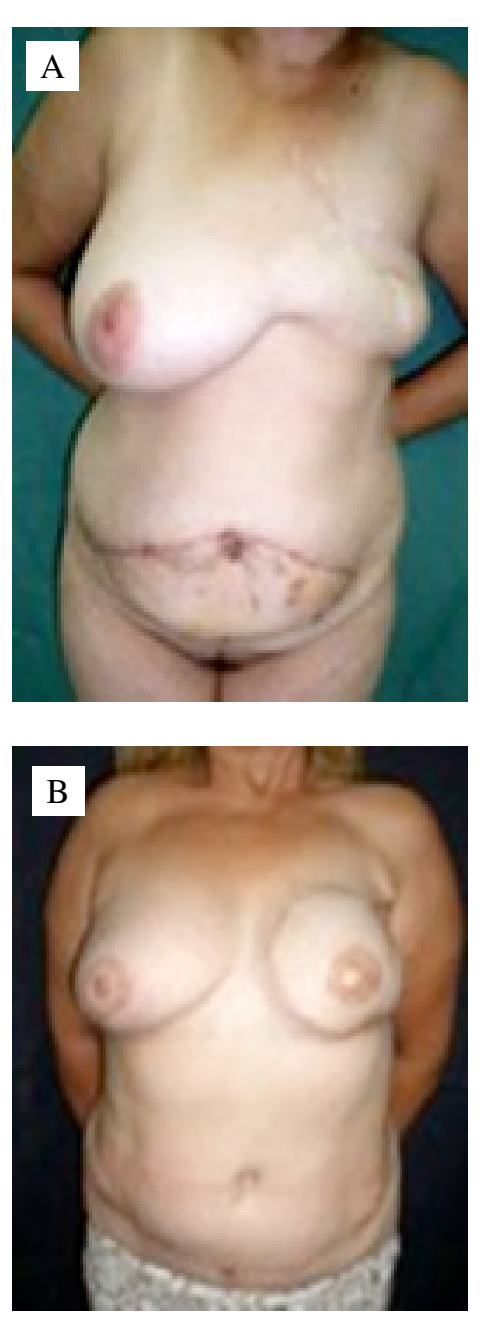

A. TRAM autonomizado (15 dias). B. Pós-operatório final (1 ano depois).

Figura 1. Retalho do músculo reto abdominal transverso autonomizado.

A autonomização (“delay phenomenon") está muito bem descrita no livro Skin Flaps. ${ }^{8-10}$ Quando realizada, faz com que a vascularização local seja mais efetiva por intermédio de uma série de fenômenos, como simpatectomia; isquemia transitória; vasodilatação e redução dos shunt artério-venosos; e liberação de inúmeros mediadores inflamatórios e fatores de crescimento. Por meio da fisiopatologia local, ocorre a vasodilatação dos vasos locais e, por consequência, do fluxo da artéria epigástrica superior, suprindo a deficiência existente. Dessa forma, a autonomização propicia uma série de fenômenos que resultam, na maioria das vezes, após período de 10 a 21 dias, no aumento da vascularização local.

Quando realizamos o TRAM em pacientes obesas e/ ou tabagistas, a diminuição da pressão sanguínea local causa maior sofrimento da nutrição celular, seja por um excesso de subcutâneo necessitando maior fluxo sanguíneo e/ou presen- 

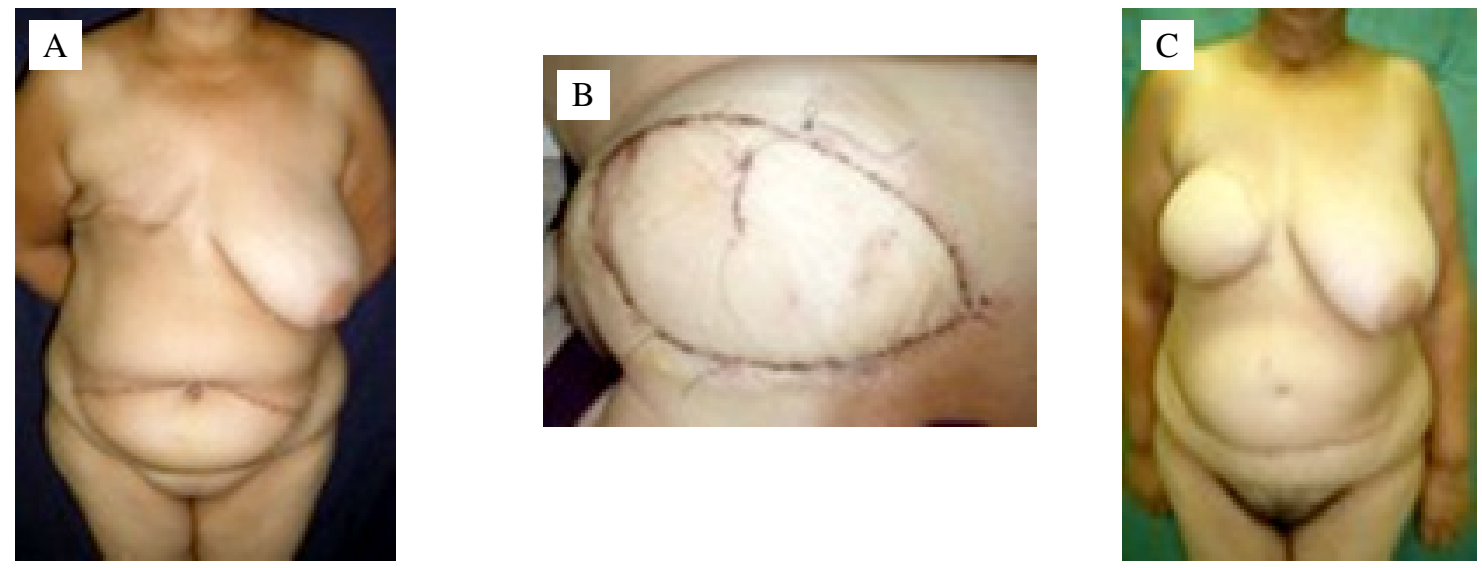

A. TRAM autonomizado (15 dias). B. Pós-operatório 15 dias após transposição. C. Pós-operatório (6 meses).

Figura 2. Retalho do músculo reto abdominal transverso autonomizado.
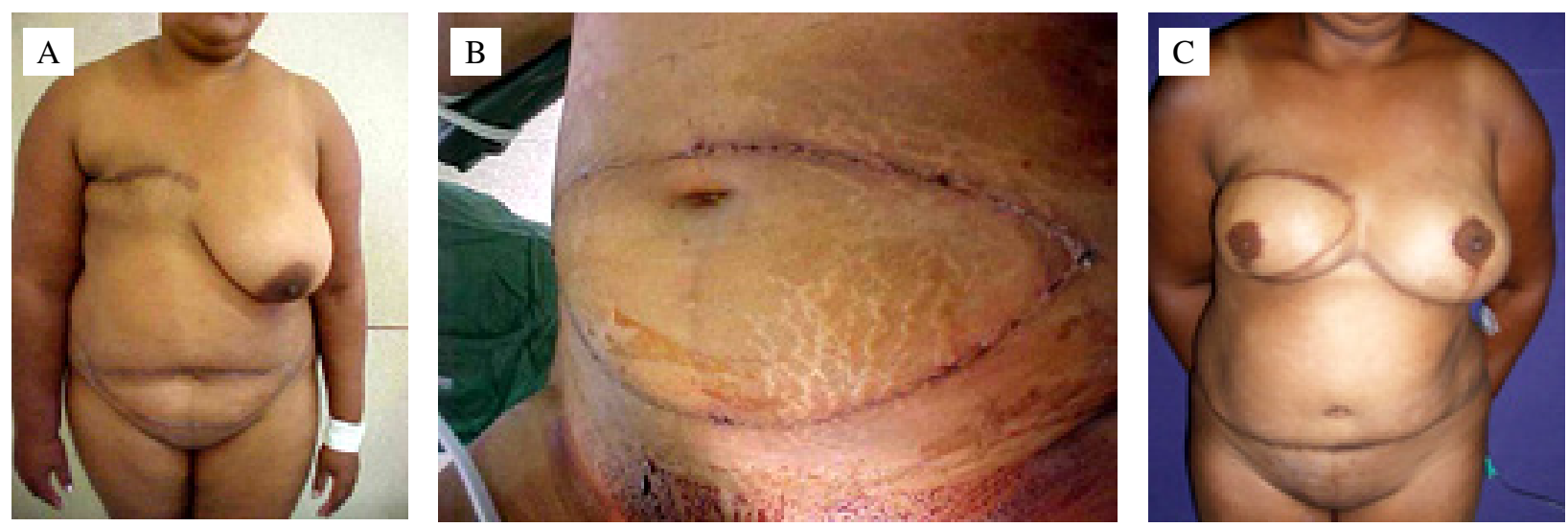

A. Pré-operatório. B. Pós-operatório (15 dias). C. Pós-operatório (2 anos).

Figura 3. Retalho do músculo reto abdominal transverso autonomizado.

ça de vasculopatia induzida pelo tabaco. Considerando um retalho miocutâneo, no qual o tecido subcutâneo é volumoso, a queda do fluxo sanguíneo não terá condições de suprir adequadamente a nutrição celular. Por mais que o organismo lance inúmeros artefatos para que isso não ocorra, fatalmente sucederá necrose parcial ou total. Portanto, a tática cirúrgica da autonomização pode provocar e estimular uma vascularização capaz de suprir e nutrir todo o retalho.

Estudos com retalhos programados para ocorrer necrose e verificar a eficácia de drogas em animais têm demonstrado eficácia em certas espécies e negativa em outras. ${ }^{8}$ Algumas drogas são eficazes em animais, não ocorrendo o mesmo em humanos. Não acreditamos que a embolização ${ }^{15}$ e a laqueadura laparoscópica ${ }^{16}$ da artéria epigástrica inferior, realizadas por alguns autores, proporcionem os fenômenos da autonomização e sejam eficazes na prevenção das necroses ao confeccionar o retalho. Principalmente porque a incisão de toda a pele e do subcutâneo na autonomização causa maior estímulo isquêmi- co e reações inflamatórias. Os autores consultados referem necrose em 13,6\% dos pacientes, sem referir obesidade ou não, e em fumantes, 16,7\%, sem comparar grupos com e sem a embolização.

Em outras épocas, a autonomização de retalhos era realizada rotineiramente. Agora, sua associação com os retalhos miocutâneos TRAM tem-se mostrado muito útil e eficaz como tática cirúrgica em pacientes com maior risco de necrose do retalho.

\section{CONCLUSÃO}

Em todos os pacientes o plexo dos vasos epigástricos inferiores foram facilmente identificados.

As táticas cirúrgicas de autonomizar o TRAM e realizar a transposição após 15 dias mostraram-se eficazes..

$\mathrm{O}$ baixo índice de complicações sugere que a autonomização do TRAM aumenta a vascularização do retalho e, consequentemente, a sua viabilidade e segurança. 


\section{REFERÊNCIAS}

1. DeBono R, Thompson A, Stevenson JH. Immediate versus delayed free TRAM breast reconstruction: an analysis of perioperative factors and complications. $\mathrm{Br}$ J Plastic Surg. 2002;55(2):111-6.

2. Ribeiro RD. Reconstrução da mama: seis anos de experiência do Núcleo de Cirurgia Plástica do Hospital Universitário Prof. Polydoro Ernani de São Thiago UFSC [trabalho de conclusão de curso]. Florianópolis: Universidade Federal de Santa Catarina; 2005.

3. Carramenha e Costa MA, Carriquiry C, Vasconez LO, Grotting JC, Herrera RH, Windle BH. An anatomic study of the venous drainage of the TRAM flap. Plast Reconst Surg. 1987;79(2):208-17.

4. Scheverien M, Saint-Cyr M, Arbique G, Brown SA. The arterial and venous anatomies of the deep inferior epigastric perforator and superficial inferior epigastric flap. Plast Reconst Surg. 2008;121(6):1909-19.

5. Mücke T, Borgmann A, Wagenpfeil S, Günzinger $\mathrm{R}$, Nöbauer C, Lange $\mathrm{R}$, et al. Autonomization of epigastric flaps in rats. Microsurgery. 2011;31(6):472-8.

6. Lopes Filho R, Silva AL. Retalho transverso músculo cutâneo do reto abdome autonomizado e transposto para o tórax: avaliação da área de necrose: estudo experimental em ratos. Rev Soc Bras Ci Plast. 2005;20(1):46-9.

7. Groth AK, Campos ACL, Gonçalves CG, Silva RFC, Silva ABS, Greca FH, et al. Effects of venous supercharging in deep inferior epigastric artery perforator flap. Acta Cir Bras. 2007;22(7):474-8.
8. Bittencourt RC, Biondo-Simões ML, Paula JB, Martynetz J, Groth A. Influence of minoxidil on ischemic cutaneous flaps in rats. Acta Cir Bras. 2005;20(6):450-4.

9. Codner MA, Bostwick J III. Delay of the TRAM flap. Oper Tech Plast Reconstr Surg. 1994;1(1):22-7.

10. Wang HT, Hartzell T, Olbrich KC, Erdmann D, Georgiade GS. Delay TRAM flap reconstruction improves flap rehability in the obese patient. Plast Reconstr Surg. 2005;116(2):613-8.

11. Myers MB. Investigation of skin flap necrosis. In: Grabb WC, Myers MB, McDowell F, editors. Skin flaps. Boston: Little Brown; 1975. p. 3-10.

12. Myers MB. Attempts to augment survival in skin flaps: mechanism of the delay phenomenon. In: Grabb WC, Myers MB, McDowell F, editors. Skin flaps. Boston: Little Brown; 1975. p. 65-79.

13. Reinisch JF. The role of arteriovenous anastomoses in skin flaps. In: Grabb WC, Myers MB, McDowell F, editors. Skin flaps. Boston: Little Brown; 1975. p. 81-92.

14. Laurent B, Rouif M, Giordano P, Mateu J. Breast reconstruction with TRAM flap after selective embolization of the deep inferior epigastric artery (series of 69 patients). Ann Chir Plast Esthet. 2011;56(6):548-54.

15. Aboutanos SZ, Spino E, Blauchet NP. Angiographic delay: a viable alternative to surgical delay. Ann Plast Surg. 2012;68(6):562-7.

16. Trus TL, Collins ED, Demas C, Kerrigan C. Initial experience with laparoscopic inferior epigastric vessel ligation for delayed TRAM flap breast reconstruction. Arch Surg. 2007;142(4):362-4. 